\title{
Complex Propagation Patterns Characterize Human Cortical Activity during Slow-Wave Sleep
}

\author{
Balázs Hangya, ${ }^{1}$ Benedek T. Tihanyi, ${ }^{1}$ László Entz, ${ }^{2,3}$ Dániel Fabó,${ }^{2,3}$ Loránd Eröss, ${ }^{2}$ Lucia Wittner, ${ }^{2,3}$ Rita Jakus, ${ }^{2}$ \\ Viktor Varga, ${ }^{1}$ Tamás F. Freund, ${ }^{1}$ and István Ulbert ${ }^{2,3,4}$ \\ ${ }^{1}$ Department of Cellular and Network Neurobiology, Institute of Experimental Medicine, Hungarian Academy of Sciences, Budapest, Hungary, ${ }^{2}$ National \\ Institute of Neuroscience, Budapest, Hungary, ${ }^{3}$ Institute for Psychology, Hungarian Academy of Sciences, Budapest, Hungary, and ${ }^{4}$ Faculty of Information \\ Technology, Péter Pázmány Catholic University, Budapest, Hungary
}

Cortical electrical activity during nonrapid eye movement (non-REM) sleep is dominated by slow-wave activity (SWA). At larger spatial scales $(\sim 2-30 \mathrm{~cm})$, investigated by scalp EEG recordings, SWA has been shown to propagate globally over wide cortical regions as traveling waves, which has been proposed to serve as a temporal framework for neural plasticity. However, whether SWA dynamics at finer spatial scales also reflects the orderly propagation has not previously been investigated in humans. To reveal the local, finer spatial scale $(\sim 1-6 \mathrm{~cm})$ patterns of SWA propagation during non-REM sleep, electrocorticographic (ECoG) recordings were conducted from subdurally implanted electrode grids and a nonlinear correlation technique [mutual information (MI)] was implemented. MI analysis revealed spatial maps of correlations between cortical areas demonstrating SWA propagation directions, speed, and association strength. Highest correlations, indicating significant coupling, were detected during the initial positive-going deflection of slow waves. SWA propagated predominantly between adjacent cortical areas, albeit spatial noncontinuities were also frequently observed. MI analysis further uncovered significant convergence and divergence patterns. Areas receiving the most convergent activity were similar to those with high divergence rate, while reciprocal and circular propagation of SWA was also frequent. We hypothesize that SWA is characterized by distinct attributes depending on the spatial scale observed. At larger spatial scales, the orderly SWA propagation dominates; at the finer scale of the ECoG recordings, non-REM sleep is characterized by complex SWA propagation patterns.

\section{Introduction}

Cortical slow-wave activity (SWA; in the $\sim 0.5-2 \mathrm{~Hz}$ frequency range) is the EEG correlate of synchronized active (up) and silent (down) states of large populations of neocortical neurons during deep nonrapid eye movement (non-REM) sleep or slow-wave sleep (SWS) (Steriade et al., 1993; Cash et al., 2009; Csercsa et al., 2010, Le Van Quyen et al., 2010). Although synchronous up and down states were observed in isolated neocortex in vitro (Cossart et al., 2003), several studies showed that the thalamus might also play an active role in shaping cortical SWA (Sirota and Buzsáki, 2005; Crunelli and Hughes, 2010; Magnin et al., 2010). Largescale thalamocortical networks were shown to engage in synchronous low-frequency oscillations (Sirota and Buzsáki, 2005; Volgushev et al., 2006). Furthermore, the hippocampus as well as subcortical centers could also participate in this process (Isomura et al., 2006; Wolansky et al., 2006; Mena-Segovia et al., 2008),

Received March 24, 2011; revised April 28, 2011; accepted May 2, 2011.

Author contributions: L. Entz, D.F., L. Erőss, R.J., and I.U. performed research. B.H., V.V., T.F.F., and I.U. designed research; B.H., B.T.T., L. Entz, and L.W. analyzed data; B.H., V.V., and I.U. wrote the paper.

This work was supported by Országos Tudományos Kutatási Alapprogramok (0TKA) PD-77864, 0TKA K-81357، Nemzeti Kutatási és Technológiai Hivatal (NKTH)-Agence Nationale de la Recherche Neurogen, NKTH-OTKA CNK77793, and National Institutes of Health Grant MH54671. L.W. was supported by Bolyai János Research Fellowship. We thank Emilia Tóth for helping us with the reconstruction of the Brodmann areas.

Correspondence should be addressed to Balázs Hangya at his present address: Cold Spring Harbor Laboratory, 1 Bungtown Road, Cold Spring Harbor, NY 11724. E-mail: bhangya@cshl.edu.

DOI:10.1523/JNEUROSCI.1498-11.2011

Copyright $\odot 2011$ the authors $\quad 0270-6474 / 11 / 318770-10 \$ 15.00 / 0$ indicating that slow oscillations could provide a general clockwork for a large variety of neural operations (Sirota and Buzsáki, 2005; Buzsaki, 2006). This view is further strengthened by a series of observations indicating that SWA is indispensable for precisely coordinating hippocampal and thalamocortical oscillations. Population activity patterns like hippocampal ripples and synchronously appearing cortical spindles are orchestrated by the cortical SWA, being entrained to the first half of the surface positive, active phase or up state of slow-wave cycles (Siapas and Wilson, 1998; Mölle et al., 2006; Clemens et al., 2007, Csercsa et al., 2010). Also, cortical SWA was shown to propagate over large distances as traveling waves (Massimini et al., 2004; Murphy et al., 2009). From another point of view, memory consolidation processes are often reflected in local changes of cortical SWA (Huber et al., 2004; Massimini et al., 2009) and asynchronies in thalamocortical slow rhythms at different recording sites were reported in some studies (Sirota and Buzsáki, 2005, their Fig. 2). Recent reports of regional and temporal heterogeneity of cortical slow waves (Mohajerani et al., 2010), as well as alternative propagation patterns such as spiral waves (Huang et al., 2010), raise the possibility that, in addition to the large-scale orderly traveling of slow waves, complex propagation patterns emerge in a temporally parallel manner at a finer spatial scale.

Signals from subdural electrodes provide substantially better spatial localization compared with scalp recordings as a result of the absence of distorting, integrating, and attenuating effects of interleaved tissues (Buzsaki, 2006; Bangera et al., 2010). These 
Table 1. Patient characteristics

\begin{tabular}{lclll}
\hline Patient ID & Age at onset (years) & Age at surgery (years) & Epileptic focus (seizure onset zone) & MRI abnormality \\
\hline Pt. 1 & 4 & 26 & Left frontobasal-temporopolar & Left temporopolar microgyria \\
Pt. 2 & 5 & 16 & Right superior frontal gyrus & Right superior frontal gyrus flair abnormality \\
Pt. 3 & 11 & 34 & Right supplementary motor area and cuneus & Negative \\
Pt. 4 & 30 & 42 & Left parietal tuber & Left temporoparietal multiplex tubers (sclerosis tuberosa) \\
Pt. 5 & 1 & 40 & Left parietal operculum and posterior part of insula & Negative \\
Pt. 6 & 1.5 & 29 & Left parietal operculum and posterior part of insula & Left parietal and insular flair abnormality \\
\hline
\end{tabular}

advantages allowed us to investigate the fine-scale $(\sim 1 \mathrm{~cm})$ propagation patterns of sleep slow waves such as convergence, divergence, reciprocal, and circular propagation. We analyzed subdural electrocorticographic (ECoG) recordings by extending classical linear correlation with information theory-based measures characterized by higher sensitivity in detecting nonlinear interactions commonly observed in neural systems (Freiwald et al., 1999). In contrast to the orderly SWA propagation patterns observed in scalp EEG recordings, we found a high prevalence of complex SWA patterns at the finer spatial scale provided by the ECoG traces. This spatial scale-dependent distinction in electrical activity patterns may reflect the different processing strategies at the local and global cortical levels during SWS.

\section{Materials and Methods}

\section{Patient selection}

Patients (Pts.) participating in this study $[n=6$, five men and one woman (Pt. 3)] had medically intractable complex partial seizures and were referred to our epilepsy surgical center for presurgical evaluation (Table 1). All patients underwent intracranial electrode implantation as required for localization of epileptogenic tissue before therapeutic resection. Patients or their legal guardians signed the informed consent form before surgery after detailed explanation of the risks. The consent forms were approved by the local ethical committee of the National Institute of Neuroscience according to the World Medical Association Declaration of Helsinki.

\section{Electrode implantation and intracranial recording protocols}

Since the noninvasive evaluation was inconclusive, all of the patients underwent subdural strip and grid electrode implantation (various subdural electrodes; distance between adjacent electrodes, $10 \mathrm{~mm}$; Ad-Tech Medical Instrument). Implantation site selection was based on results of previous noninvasive clinical studies for seizure focus localization. We used standard craniotomies to insert the electrodes into the subdural space under general anesthesia. The electrode cables were tunneled under the skin to avoid infection and the bone flap was cryopreserved for delayed reimplantation to avoid any complication from significant brain swelling. A postimplantation MRI (3D sequence, $1 \mathrm{~mm}$ slice thickness) was routinely performed to visualize the final position of the electrodes. After 3-dimensional reconstruction and skull stripping of the images (Brain Extraction Tool), electrode contacts were individually localized with the aid of intraoperative photographs.

After recovery from surgery, the patients were admitted to the epilepsy monitoring unit and were continuously monitored for seizures. VideoEEG monitoring was performed using a 128 channel Brain Quick System 98 (Micromed). All signals were recorded to a mastoid reference (acquisition rate: 1024 or $512 \mathrm{~Hz}$, filtered between 0.1 and $250 \mathrm{~Hz}$ ).

\section{Slow-wave sleep detection}

Since the sleep of the patients was fragmented due to medical care and distress from the hospitalization and head wound, we cannot provide the standard hypnograms that are usually obtained from subjects undergoing standard polysomnography studies without recent craniotomy. Craniotomies may also distort the scalp distribution of the EEG due to lack of bone and excessive fluid accumulation below the scalp. Furthermore, scalp electrodes placed close to frontal craniotomy wounds may induce infection; therefore, we avoided placing more than two frontal scalp EEG electrodes. In fact, we attempted to use these scalp EEG elec- trodes whenever possible to aid our partial sleep staging method, but most of the time their signal was not adequate for this purpose because of the desiccation of the EEG electrode gel and other reasons causing large and variable electrode impedances, electromagnetic interference, and mechanical artifacts. To overcome these hardships, our partial sleep staging method in the clinical environment was based on the recordings from frontal ECoG channels and long-term video, without measures like EMG, EOG, ECG, and respiration monitoring that are usually recorded with EEG in a standard polysomnography environment. Behavioral sleep was confirmed by the all night, long-term video recording, while deep non-REM sleep periods were electrographically identified using frontal ECoG traces by expert neurologists. In particular, deep non-REM sleep periods (or SWS) were identified when a 30-s-long sleep epoch contained $>20 \%$ of slow waves with $>75 \mu \mathrm{V}$ peak-to-peak amplitude falling in the $0.5-2 \mathrm{~Hz}$ frequency range. The above criteria are consistent with the staging criteria of N3 or SWS, according to the recent American Academy of Sleep Medicine guidelines (Iber et al., 2007). In our previous work, using similar partial staging techniques based on ECoG traces and video records, we were able to clearly distinguish between light and deep non-REM sleep (Cash et al., 2009, their Fig. $2 B, C)$. In another study using similar staging methods, we were also able to clearly detect the typical SWA pattern in SWS demonstrated in previous animal and human studies (Csercsa et al., 2010, their Fig. 4B, supplemental Fig. 1). Data containing interictal spikes (within $1 \mathrm{~min}$ ) and seizures (within overall $60 \mathrm{~min}$; $40 \mathrm{~min}$ in Pt. 4) were excluded from the study to avoid epileptic contamination.

\section{Data analysis}

Data preprocessing. Subgrids of $4 \times 4$ (Pts. 1, 3, and 5) or $4 \times 5$ (Pts. 2, 4 , and 6) electrodes were selected for analysis (Fig. 1A). The reduction of the number of grid electrodes was necessary to analyze similar number of channels for comparability reasons and to reduce computational time. Selection of subgrids was based on grid location [an attempt was made to include frontal electrodes where SWA is typically more pronounced (Kurth et al., 2010)] and recording quality (channels not functioning were excluded). For each patient, three to five 1-min-long segments (26 segments in total) of normal SWS were selected. Principles of segment selection were to avoid epileptic contamination (see below) and to include deep-sleep epochs with prominent SWA. In Pt. 6, all segments were recorded in a single night, whereas in the other patients, segments from different nights were also included. Selected segments were preceded and followed by $30 \mathrm{~min}$ of seizure-free activity (except Pt. 4, where it was preceded by $30 \mathrm{~min}$ and followed by $10 \mathrm{~min}$ ) and were free from interictal spikes. ECoG traces were resampled at $1000 \mathrm{~Hz}$ and bandpass filtered $(0.1-40 \mathrm{~Hz})$ with a finite impulse response filter using zero phase shift filtering (fir1.m and filtfilt.m built-in Matlab functions) to remove highfrequency ECoG components and electrical noise.

Calculation of propagation maps. We assessed associations between ECoG traces from different channels using mutual information (MI). MI provides the amount of information shared by two variables and is considered a nonlinear measure of covariation (Na et al., 2002; Kajikawa and Hackett, 2005; Hangya et al., 2009). The choice of MI instead of linear correlations was motivated by its ability to detect any kind of dependency without assumptions about the structure of correlations or the underlying distributions (Na et al., 2002; Kajikawa and Hackett, 2005; Paz et al., 2010), which is important because nonlinear correlations are generally common among neural signals (Freiwald et al., 1999; Hangya et al., 2009). We further justified this choice by comparing MI with linear cross-correlation analysis (see Results, below) (Fig. 2). 
For each 1000 ms segment, ECoG values were binned into fixed bins between the overall minimal and maximal value. MI was calculated between different ECoG channels using the classic formulation in combination with Panzeri-Treves bias correction (Shannon, 1948; Panzeri et al., 2007), as described previously (Hangya et al., 2009). The basic idea of timeshifted correlations or cross-correlations was applied (Ostojic et al., 2009), using MI instead of linear correlation, as follows. On each channel, 1-s-long ECoG segments $\left(X_{\mathrm{t}}\right.$ to $\left.X_{\mathrm{t}+1000}\right)$ were correlated to later segments from all other electrodes separately $\left(Y_{\mathrm{t}+\tau}\right.$ to $Y_{\mathrm{t}+\tau+1000}, 0<$ $\tau<1000 \mathrm{~ms}$ ) by calculating MI (temporal resolution, $1 \mathrm{~ms}$ ) (Fig. $1 B$ ). Maximal MI in the function of the time lags $(\tau)$ was calculated for all time points $(t)$ and channel pairs. The choice of 1-s-long time windows was motivated by the observation that SWA frequency is around or below $1 \mathrm{~Hz}$ (Crunelli and Hughes, 2010; Csercsa et al., 2010; Diekelmann and Born, 2010), thus these time windows were able to approximately include a slow-wave cycle. However, it has been shown that MI calculations are robust in the selection of the time windows (Paz et al., 2010). The above calculations were repeated for overlapping 1-s-long ECoG segments (900 ms overlap). Next, correlations were destructed by cutting the ECoG data to 1-1.2-s-long segments, which were then shuffled using the randperm.m built-in Matlab function, which randomly permutes integers based on a uniform distribution. Permutation was repeated if a data segment remained in its original position. Significance of maximal MI was tested by comparing it to a distribution of MI values calculated from such shuffled versions of the original data (number of shuffles ranged from 151,296 to 236,400). A significance level of $p=0.0001$ was used to reduce the number of false-positive detections according to high numbers of statistical tests (this choice corresponds to approximately one false detection in $2.5 \mathrm{~s}$ ). In case a significant maximal MI was found, waveform correlation was established between the two channels at time point $t$. It should be noted that according to the binning between the minimal and maximal data values, MI reflected predictability between slow-wave cycles rather than higher frequency (lower amplitude) oscillations such as spindles (see Results, below). Thus, significant maximal MI showed the propagation of SWA between two cortical areas, the temporal maximum location provided propagation time, and the maximal MI value indicated correlation strength. Propagation speed was calculated by dividing electrode distances by propagation time values. Time-resolved propagation maps were created using the above parameters, visualized as movies and further analyzed (see below). Unitary propagation events (Fig. $1 C$, bottom left, arrows) were assessed by significant propagation between two ECoG channels. Start and end points of such events were defined by the zero $(t)$ and maximal location $(t+\tau)$ points in the time lag-MI plot (Fig. $1 B$, right). According to the overlap-
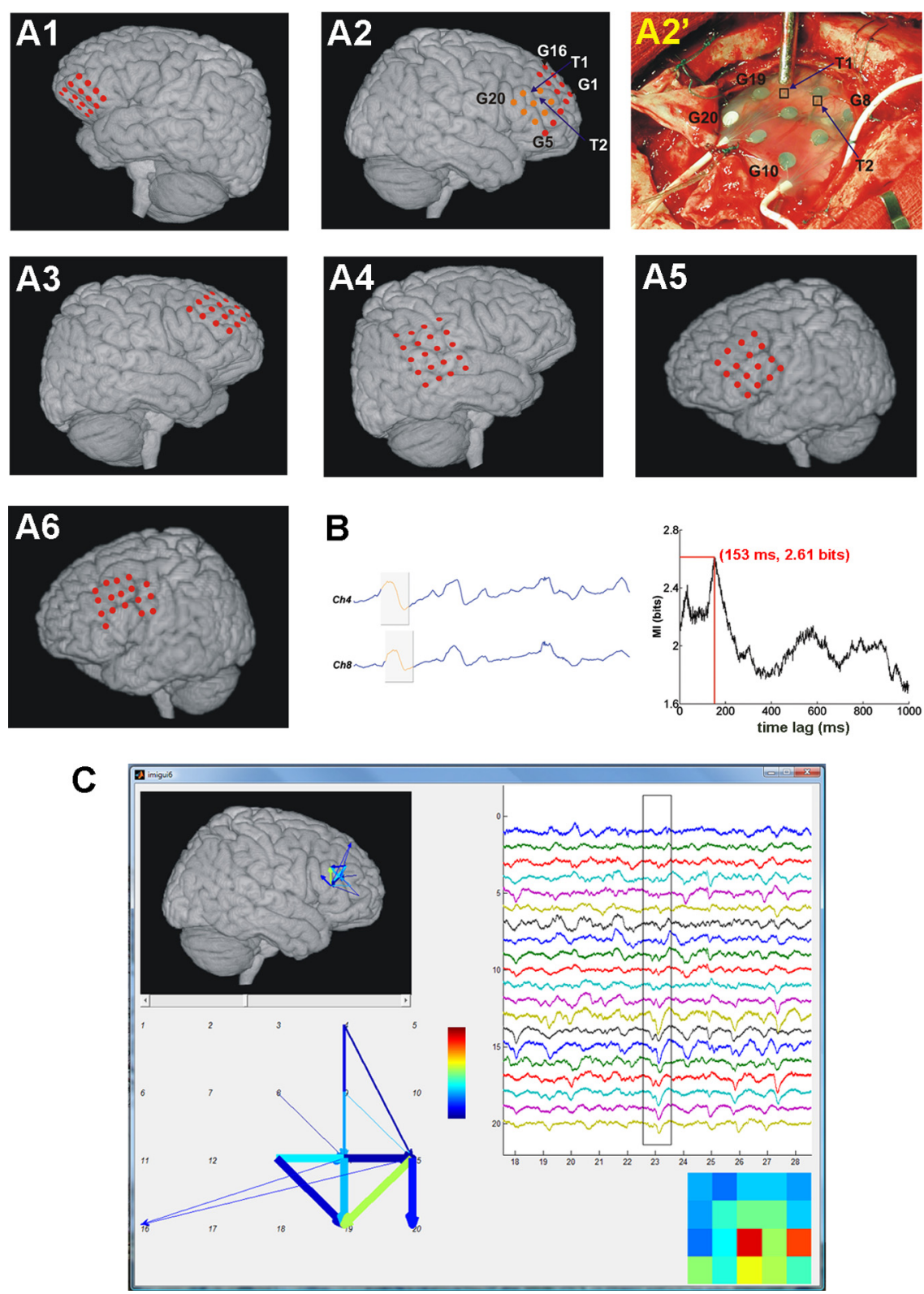

Figure 1. Recording and analysis of SWA propagation. $A$, Estimations of the positions of recording sites on standard human brain images based on intraoperative photographs and postoperative structural MR images (A1-A6 correspond to Pts. 1-6, respectively). $\mathbf{A} \mathbf{2}^{\prime}$, Photograph taken during the operation. Marked points and electrodes are also exhibited in the corresponding reconstruction image (A2). Recording sites visible in $\boldsymbol{A 2}$ ' are displayed in orange in $\mathbf{A 2}$. Channel $18 \mathrm{in} P$ t. 2 as well as channels 5, 17, and 18 in Pt. 6 were dysfunctional (i.e., either no data were recorded by the electrode, or a disproportionate amount of nonbiological noise showed the malfunction of the contact site) and thus left out from the analysis. $\boldsymbol{B}$, Filtered $(0.1-40 \mathrm{~Hz}) \mathrm{ECOG}$ data were subjected to Ml analysis for all pairs of recording channels (see Materials and Methods). One-second-long data segments were compared with time-lagged data from the other channel and the extent of nonlinear correlation (predictability) was measured by MI. Left, Short data segments from two ECoG channels (Ch). Gray shading designates examples of 1-s-long temporal windows for MI calculations. Right, MI was displayed as the function of time lags (1 ms resolution). A significant maximal MI (in this case, 2.61 bits at 153 ms temporal delay) was considered to be a sign of significant correlation between the two channels, which corresponded to propagating sleep slow waves (see Materials and Methods and Results). Left, Time lag between the data windows (gray) was set to the optimal delay (153 ms) at the given time point. Note the similarity of slow-wave cycles (orange) in the windows. This analysis was repeated for all fixed time instances and for all pairs of recording channels. C, Based on the Ml analysis, SWA propagation time, distance, and association strength could be assessed for all significant unitary propagation events, from which a time-resolved propagation map was drawn and visualized in a custom-built graphical user interface. A screenshot from this interface is shown with all ECOG channels (top right), amplitude map [bottom right; coldest/warmest color correspond to smallest/largest ECOG amplitude (color bar); $5 \times 4$ squares correspond to the $5 \times 4$ grid electrodes; amplitude was calculated as the difference of maximal and minimal $E C O G$ value in the data window], propagation map displayed in a schematic form (bottom left) and also projected to the brain surface (top left). For every time point, arrows on the propagation map designate unitary propagation events between two recording sites (marked by numbers on the schematic view). The color of each arrow shows association strength (warmer colors are stronger) and the width corresponds to propagation time (thicker lines show shorter time). 
A

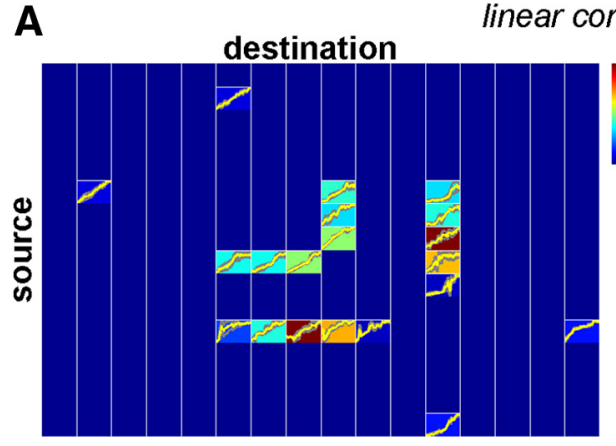

linear correlations
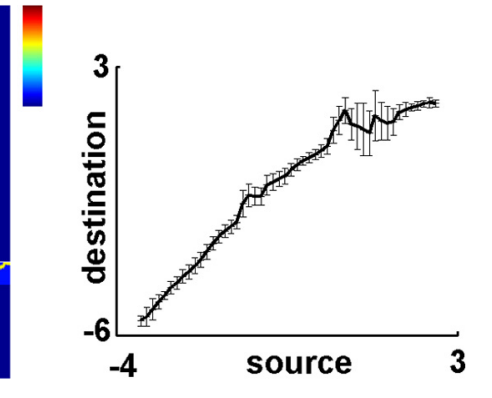

B

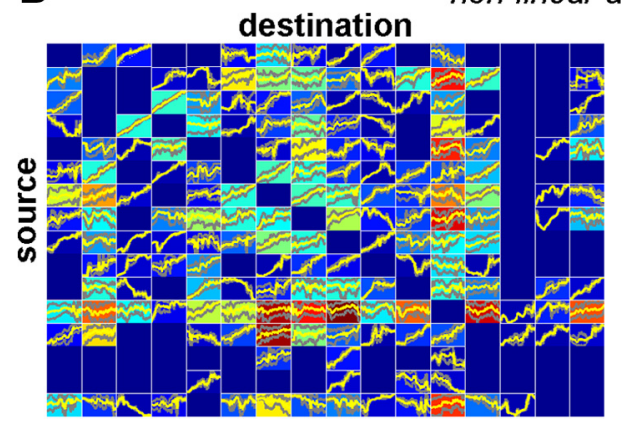

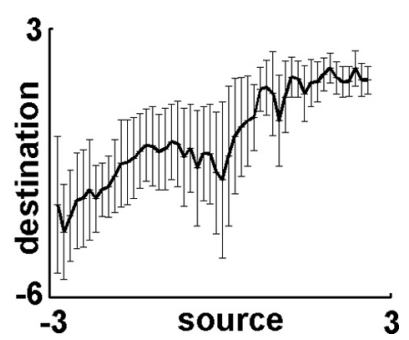

3

Figure 2. Nonlinear associations during SWA propagation. $\boldsymbol{A}$, Left, Correlation profile and input-output curves of linear crosscorrelation analysis from a representative subject. Each colored field corresponds to a pair of recording channels ( $y$-axis, source channel; $x$-axis, destination channel). Colors indicate the total strength of (significant) directional correlation between the source and destination channels (i.e., significant cross-correlation with a positive time lag when calculated with reference to the source channel; warm colors are high correlation values) (for significance testing of linear and nonlinear correlations, see Materials and Methods). A significant cross-correlation between two channels at $\tau$ milliseconds means that the ECoG segment of the source channel is correlated with that of the destination channel shifted by $\tau$ milliseconds. We collected these correlated pairs of data segments for each pair of channels and plotted the ECOG values of the destination channel against corresponding values of the source channel (binned and averaged). These input- output functions are plotted in the colored fields for all channel pairs (yellow, mean; gray, SD). Right, A typical input- output function (source, channel 8; destination, channel 9) is enlarged. Error bars indicate SD. ECOG segments were standardized (i.e., transformed to zero mean and unitary SD); thus, tick labels on both axes show standardized voltage values. Note the line-like shape of these input-output curves. $\boldsymbol{B}$, Left, Nonlinear correlation profiles and input- output curves for the same sleep segment calculated by the MI method. Arrangement follows that of $\boldsymbol{A}$. Right, An example input- output function (source, channel 2; destination, channel 11) is enlarged. Note the higher abundance of significant nonlinear associations, the moderate similarities of linear and nonlinear correlation profiles (e.g., low rate/absence of correlations for channels 14 and 15 , strong correlation between channels 8 and 12), and the more complex and variable shape of nonlinear input- output curves. Higher SD values and noisier appearance of the nonlinear input- output functions show that the nonlinear correlation functions between pairs of channels exhibit temporal changes.

ping windows (see above), the time resolution of propagation map frames was $100 \mathrm{~ms}$, whereas the temporal resolution of time delays was 1 $\mathrm{ms}$ (see above). Consistency of time delays for direct and indirect associations was checked to test the robustness of the method.

Correlation triggered average. To visualize ECoG patterns giving rise to significant correlations, we calculated ECoG averages triggered by significant MI values (Fig. 3A). That is, if a significant MI was detected at a certain time, a 1-s-long ECoG segment centered to that time point was stored. At the end, these segments were averaged for each recording channel. The average signal reflected typical ECoG patterns underlying significant correlations.

Analysis of propagation patterns. Convergence/divergence of SWA was defined as unitary propagation events ending/starting at the same recording site within a $100 \mathrm{~ms}$ time window. Divergence maps were smoothed over two consecutive frames because of the discrete nature of the starting points of unitary propagation events. Two-dimensional distributions showing relative contribution to the convergence/divergence at a single location were termed "contribution maps." Cortical centers with high convergence/divergence rate did not show correlation with epileptic foci. Reciprocal connections were defined as reciprocal propagation between two sites within $100 \mathrm{~ms}$. Three-point circles (i.e., directed three-point trajectories of which the end points are the same as the starting points) were defined in an analogous way. Controls for the above measures were generated by within-frame random shuffling (uniform distribution using randperm.m built-in Matlab function) of unitary propagation events among the electrode pairs. Control distributions for reciprocal connections and threepoint circles were generated by 250 or 1000 shuffles. If the value derived from the real data was out of the entire range of control distributions, we used the $\mathrm{p} \approx 0$ notation, which can be translated to at least $p<0.004$. Normalization with individual control distributions (Figs. 4, 5) was performed by subtracting the mean and dividing by the $\mathrm{SD}$ of the corresponding control distribution. The application of this procedure results in standardized control distributions and allows the comparison across subjects. Significance of correlations among slow-wave propagation characteristics, convergence, divergence, and contribution maps was assessed using $F$ test. Statistical tests were judged at a significance level of $p=0.05$ unless stated otherwise. We performed a signal-to-noise ratio analysis that confirmed that potential differences in recording quality did not have a significant effect on the propagation map evaluation (data not shown).

Linear cross-correlations. Linear crosscorrelation analysis was conducted by replacing time-shifted mutual information values with linear cross-correlations. To facilitate the comparison of the results of linear and nonlinear analyses, all other parameters of the calculations were left unchanged (including significance testing using shuffled data; see above). Thus, a directional correlation was established when a significant cross-correlation with a positive time lag was observed (positive and negative correlation were not differentiated to allow the comparison with the MI measure). We also calculated the input-output functions related to significant linear (crosscorrelation) and nonlinear (MI) correlations. For each pair of recording channels, we collected pairs of ECoG segments where a significant association was found, and plotted average ECoG amplitude values of a recording channel as a function of binned ECoG values of the other channel.

All data analysis procedures were implemented in Matlab development environment (MathWorks) using custom-built and built-in functions.

\section{Results}

Nonlinear associations during human SWS

The nonlinear nature of neural communication was shown in several systems (Freiwald et al., 1999; Darvas et al., 2009; Chen et al., 2010); however, it is unclear whether such nonlinearities also appear in the course of slow oscillation propagation. We investigated 1-min-long segments of SWS in six epileptic patients. ECoGs from 16-20 subdural recording sites were analyzed in each patient. We first searched for associations between ECoG waveforms recorded from different brain areas and compared the results of a linear (cross-correlation) and a nonlinear (MI) correlation analysis (see Materials and Methods, above).

Both linear and nonlinear types of dependencies were present in ECoG channels in all analyzed sleep segments. Significant linear correlation and MI values were summed over time for each 
pair of recording channels for each recording segment, resulting in linear and nonlinear correlation profiles. Although some similarities between the two types of profiles were discoverable (Fig. 2), significant nonlinear correlations were more abundant [5687 of 7266 channel pairs in all recordings $(78.3 \%)$ compared with 1292 of $7266(17.8 \%)$ ], showing additional associations compared with the linear analysis. It should be noted however, that linear correlations were detected in a very few cases where nonlinear correlations were not (36 of 7266 channel pair comparisons, $0.5 \%$ ), showing that in cases of pure linear associations, crosscorrelation analysis can be more sensitive than MI.

To visualize the associations between channel pairs, we calculated the input-output functions related to significant linear and nonlinear correlations. If all such inputoutput curves are line-shaped even for the MI calculations, then nonlinear analysis is unnecessary and the cross-correlation technique is sufficient to detect all associations. As expected, cross-correlations mainly captured linear relationships (Fig. 2A). However, we found that input-output curves were highly complex and often nonlinear in a number of cases for MI analysis (Fig. 2B). Thus, as nonlinear associations were present during human SWS, the MI analysis was more appropriate in characterizing slow oscillation propagation.

\section{Characteristics of SWA propagation}

The significant correlation of time-shifted waveforms recorded from pairs of electrodes was established by the MI technique. The resulting associations at each time point (Fig. $1 B, C$ ) served as unitary events for the subsequent calculations.

First, we investigated what characteristic patterns in the ECoG traces gave rise to significant waveform correlations. Raw ECoG segments showing significant nonlinear correlation with data segments recorded from other electrodes were averaged for each recording site (see Correlation triggered average, above). Significant waveform correlations usually corresponded to the rising phase of cortical SWA (Fig. $3 A$ ), which further supports the view that waveform correlations detected by the MI method reflect slow-wave propagation between different cortical areas. Moreover, it implies that the most conservative segment of the slow-wave cycle is its ascending phase, showing more pronounced correlation across areas compared with other phases of the oscillation.

Next, we calculated general characteristics of SWA propagation. The distribution of propagation distance (Fig. 1C, length distribution of the arrows) showed that SWA correlations were most common among neighboring cortical areas, although associations between distant sites were also observed (Fig. 3B). These unexpected spatial noncontinuities, or jumps, of SWA do not fit the general idea of traveling waves, i.e., propagation of slow waves as single lines over large cortical areas (Massimini et al., 2004). Propagation speed of cortical slow waves (calculated by dividing electrode distances with propagation time values; see Materi-
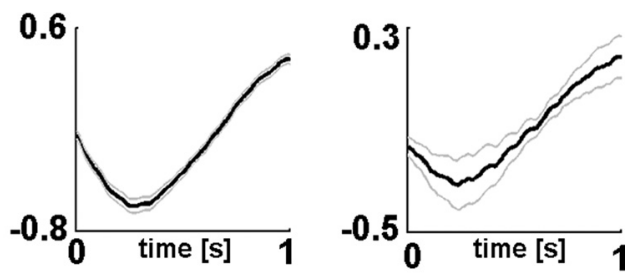

C

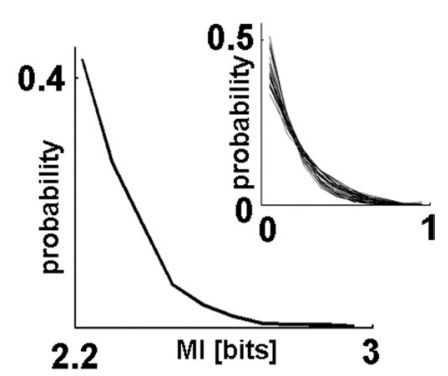

Figure 3. SWA propagation characteristics. $A$, Average waveforms of data segments with significant nonlinear correlations across $\mathrm{ECOG}$ channels. Left, Mean waveform from all ECoG channels separately (gray) and averaged (black) from a representative sleep segment of a . Only data segments that showed significant correlations with other recording channels were included in these calculations. Graylines showSEM. Right, Grand average from all patients (black, mean; gray, SEM across patients). It should benoted that EC OG segments including the rising phase of SWA. B. Propagation distance distributions of unitary propagation events (Fig. 1C, length distribution of single

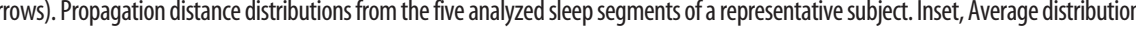
distant loci ( $p=7.12 \times 10^{-21}$, Mann-Whitney $U$ test); however, spatial noncontinuities of SWA propagation between areas located 5

als and Methods, above) showed large variability with an overall mean \pm SEM of $3.33 \pm 0.96 \mathrm{~m} / \mathrm{s}$, which is approximately in agreement with previous studies (Massimini et al., 2004; Hill and Tononi, 2005; Murphy et al., 2009). The distribution shape of correlation strength (MI) showed marked similarity across recording segments and subjects (Fig. 3C).

These propagation statistics were not statistically independent. Specifically, weak but significant negative correlations were found between correlation strength and propagation time [significant correlation $(p<0.01)$ in 24 of 26 sleep segments with a mean \pm SEM correlation coefficient of $-0.17 \pm 0.01]$ as well as propagation distance [significant correlation $(p<0.01)$ in 22 of 26 sleep segments with a mean correlation coefficient of $-0.16 \pm$ 0.01 ], whereas it was positively correlated with propagation speed [significant correlation $(p<0.01)$ in 19 of 26 sleep segments with a mean correlation coefficient of $0.10 \pm 0.01$ ]. Thus, fast propagation to short distances was accompanied by higher correlation strength. Expectedly, propagation time was usually positively correlated with propagation distance [positive correlation $(p<$ 0.01 ) in 23 of 26 sleep segments with a mean correlation coefficient of $0.28 \pm 0.02$; negative correlation in one and no significant correlation in two cases].

\section{Complex patterns of SWA propagation}

Sleep slow waves detected using scalp EEG recordings were shown to propagate along anteroposterior lines, sweeping across large areas of the cortical mantle (Massimini et al., 2004). The high signal quality of ECoG recordings (Buzsaki, 2006) allowed us to investigate SWA propagation at a finer scale. We tested 
A

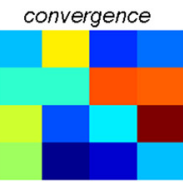

Pt.2

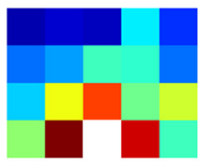

Pt.3
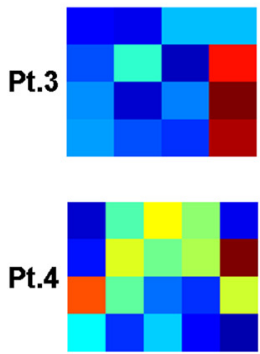

Pt.5

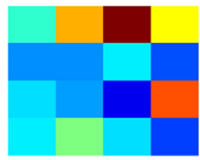

Pt.6
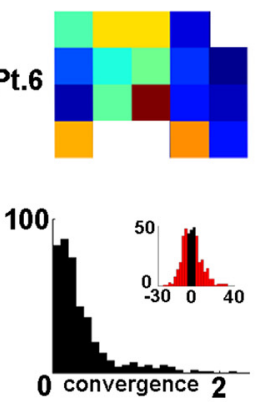

B
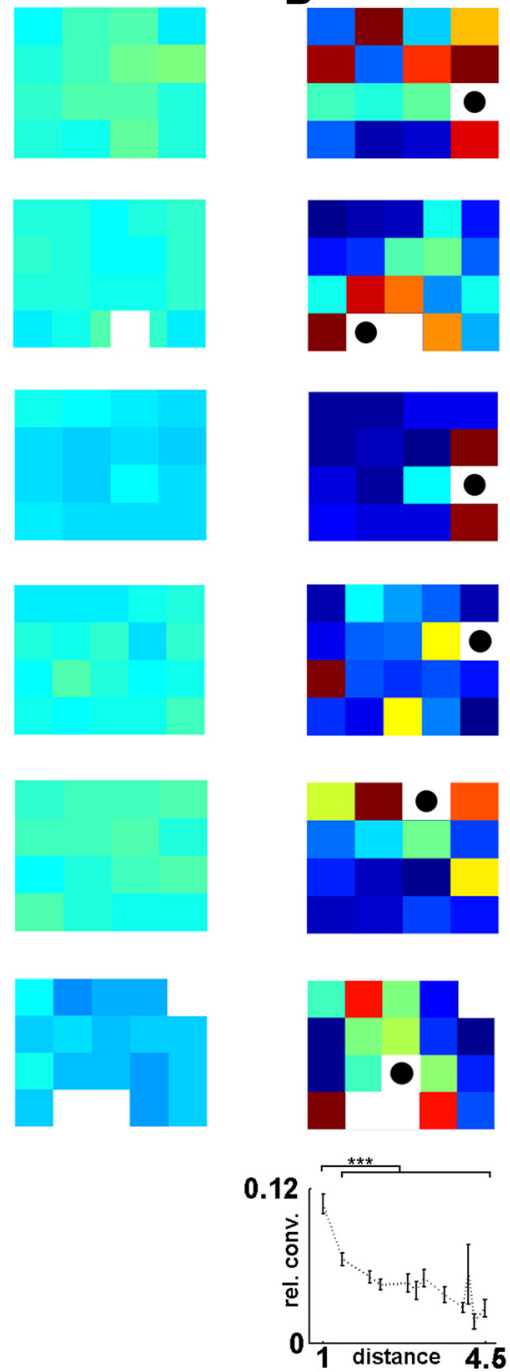

C

E
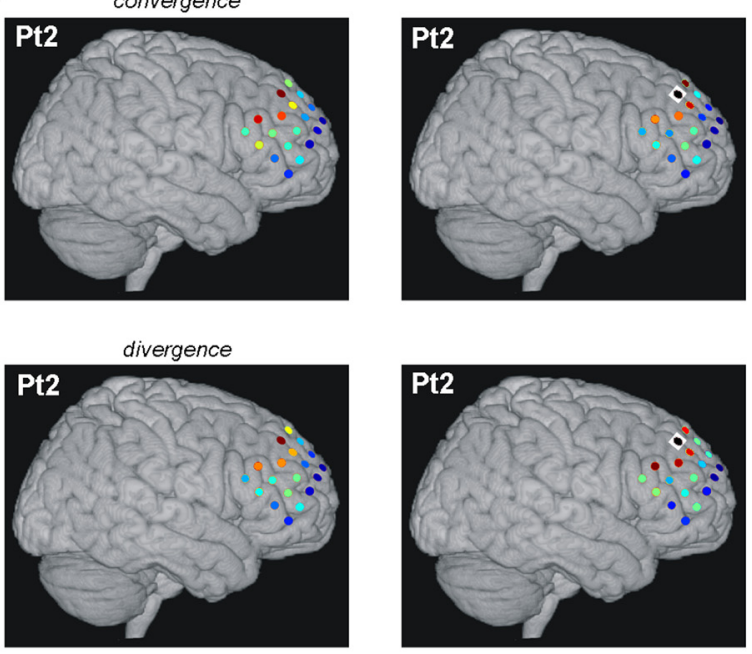
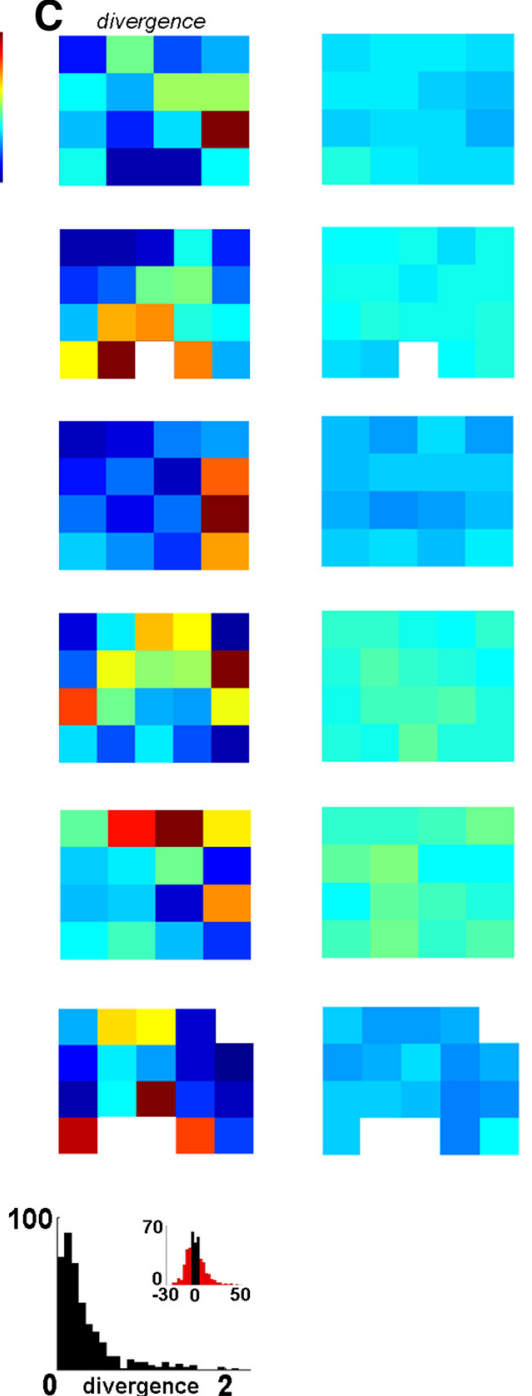

D
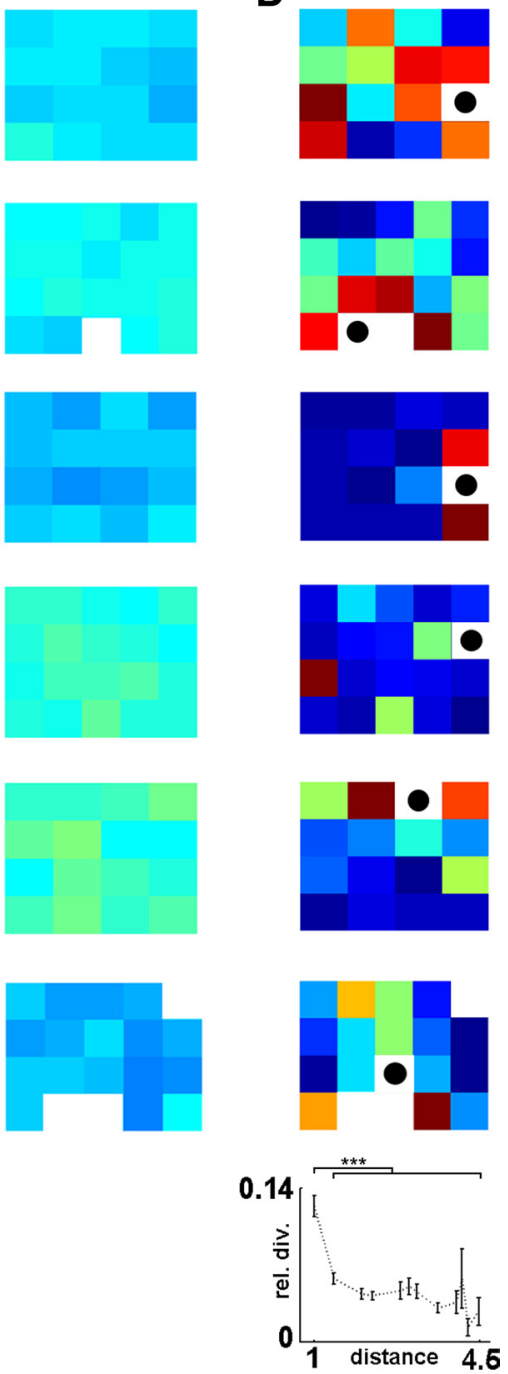

$F$
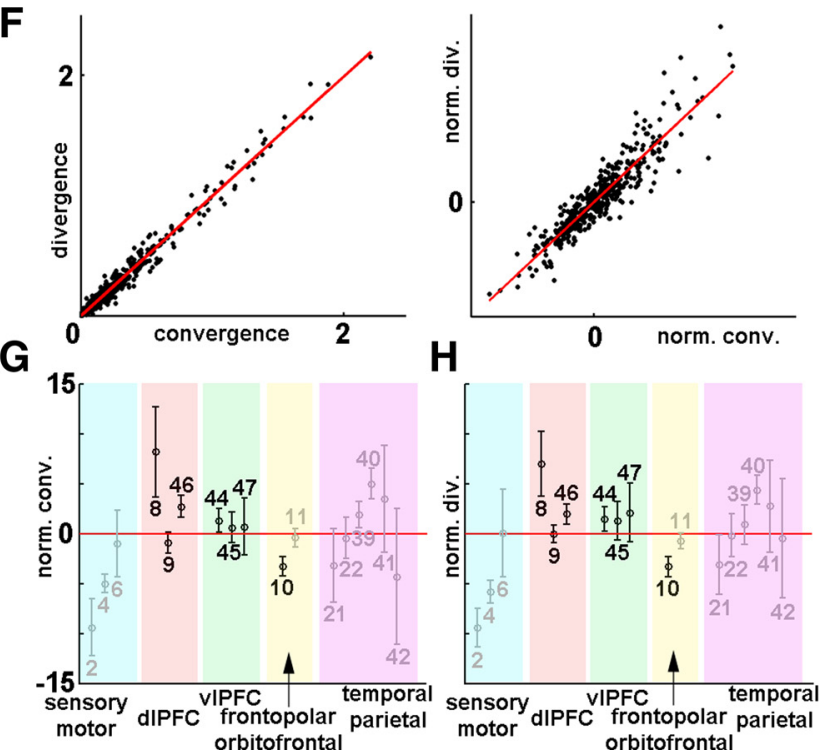

Figure 4. Convergence and divergence of SWA. A, Rate of SWA convergence on different electrodes. Warm colors indicate high number of convergent inputs (color bar). The $4 \times 4$ or $4 \times 5$ arrangements correspond to the configuration of the electrode grids (Fig. 1A). Left, Representative sleep segments from all subjects; right, corresponding control maps of randomized data (unitary propagation events were randomly shuffled among electrode pairs within each time frame, see Materials and Methods). Bottom, Distribution of the mean number of convergent inputs for all channels of all subjects. Inset, Distribution of the same values but normalized with individual control distributions (see Materials and Methods). Red bars fall out of the range of control distributions. B, Contribution maps for recording sites with the highest convergence rate (black dot in white field). Warm colors indicate high proportion of contribution to the SWA (Figure legend continues.) 
whether more localized propagation patterns merely reflect linelike spreading found at larger scales, or whether a more complex propagation pattern characterizes smaller cortical regions.

First we analyzed convergence and divergence patterns. Convergence was assessed when an area received cortical activity from at least two distinct sites within a $100 \mathrm{~ms}$ time window, a temporal frame that allows different types of synaptic plasticity (Caporale and Dan, 2008). Locations with a high occurrence of convergent activity were observed in all subjects (see convergence maps in Fig. $4 A, E)$. Sites of high convergence were preserved over different periods of SWS during the same night ( $n=6$ subjects) and also, albeit to a lesser extent, different nights (significant correlation of convergence maps were found in all subjects in 13 of 16 within-night and 13 of 30 between-night comparisons; correlation coefficient for significant within- and between-night comparisons of $0.70 \pm 0.05$ and $0.65 \pm 0.04$, respectively) (Fig. $5 A$ ). Recorded cortical areas contributed differentially to all convergent inputs to these sites (see contribution maps in Fig. 4B). These contribution maps were also statistically similar within subjects for sleep segments recorded during the same or different nights (significant correlations of contribution maps for the cortical site with the highest rate of convergence were found in all subjects in 12 of 16 within-night and 16 of 30 between-night comparisons; correlation coefficient for significant within- and between-night comparisons of $0.67 \pm 0.05$ and $0.70 \pm 0.04$, respectively) (Fig. 5A). Areas with high rate of convergence received activity mostly, but not exclusively, from neighboring cortical locations (Fig. 4B).

Divergence of SWA was defined as propagation to different cortical areas from a single recording site within a $100 \mathrm{~ms}$ time window. Divergence maps showed hot spots with high divergence rates in all subjects (Fig. 4C,E) and were similar across different sleep segments of the same night as well as across different nights within subjects (significant correlation of divergence maps were found in all subjects in 11 of 16 within-night and 12 of 30 between-night comparisons; correlation coefficient for significant within- and between-night comparisons of $0.75 \pm 0.05$ and $0.66 \pm 0.04$, respectively) (Fig. $5 B$ ). Moreover, these maps were markedly similar to the corresponding convergence maps of the same segment (significant correlation in all cases, $n=26$; correlation coefficient of $0.93 \pm 0.01$ ) (compare Fig. $4, A$ and $C$; see

$\leftarrow$

(Figure legend continued.) convergence. Maps in the same row correspond to the same sleep segment. Bottom, Group results showing relative contribution to the convergence on the most prominent convergence center in the function of propagation distance (mean \pm SEM; only distance values with at least four sample elements are shown to allow reliable statistical estimates). Adjacent cortical areas showed significantly larger relative contribution $(p=1.07 \times$ $10^{-15}$, Mann-Whitney $U$ test), although distant sites could also contribute substantially (see Pts. 1 and 4). C, Rate of SWA divergence for all electrodes. Warm colors indicate high number of divergent outputs. Arrangement follows that of $\boldsymbol{A}$. Right, Control maps. Note the similarity with corresponding convergence maps $(\boldsymbol{A})$. $\boldsymbol{D}$, Relative distribution of divergent outputs from sites with highest divergence rates. Arrangement follows that of $\boldsymbol{B}$. Diverging SWA propagated dominantly to adjacent loci $\left(p=2.07 \times 10^{-20}\right.$, Mann-Whitney $U$ test $)$, but distant areas also received substantial diverging SWA (see Pts. 1, 4, and 6). $\boldsymbol{E}$, The four maps of Pt. 2 shown in $\boldsymbol{A}-\boldsymbol{D}$ are projected onto the brain surface (left, convergence and divergence map; right, corresponding contribution maps). $\boldsymbol{F}$, Left, Convergence and divergence rates are plotted against each other for all recording sites and sleep segments in all patients. Right, The same rates were normalized to individual controls. Convergence and divergence rates were strongly correlated ( $r=0.988$ and $r=0.921$ in the raw and normalized cases, respectively; $\mathrm{p} \approx 0$ in both cases). $\boldsymbol{G}, \boldsymbol{H}$, Distribution of convergence (conv., $\boldsymbol{G}$ ) and divergence (div., $\boldsymbol{H}$ ) rates [normalized (norm.) to controls] over different Brodmann areas. Mean \pm SEM values are displayed for each Brodmann area (numbers). Black, Regions covered in at least two patients; gray, areas covered in only one patient. dIPFC, Dorsolateral prefrontal cortex; VIPFC, ventrolateral prefrontal cortex. also Fig. 4F). Thus, convergence and divergence maps changed in a correlated fashion across different nights (Fig. 5). Maps showing relative distribution of divergent outputs from these hot spots of SWA propagation (Fig. 4D) were preserved among recording segments (significant correlations were found in all subjects in 12 of 16 within-night and 18 of 30 between-night comparisons; correlation coefficient for significant within- and between-night comparisons of $0.71 \pm 0.05$ and $0.69 \pm 0.03$, respectively) (Fig. $5 B$ ) and were similar to corresponding convergence contribution maps (significant correlation in 25 of 26 cases; correlation coefficient of $0.87 \pm 0.02$ ) (compare Fig. $4, B$ and $D$ ).

The placement of the grids was determined by clinical demands resulting in only a partial overlap among patients (Fig. $1 \mathrm{~A})$. Although this arrangement prevented detailed anatomical localization of convergence and divergence centers and the construction of topographic maps for convergence and divergence rates, we nevertheless analyzed the spatial organization of these activities according to Brodmann areas (BA). BA8, BA9, and BA46 (dorsolateral prefrontal cortex); BA10 (frontopolar cortex); and BA44 and BA45 (ventrolateral prefrontal cortex; Broca area in Pts. 5 and 6 , where the grid was above the left hemisphere) were partially covered by the grid in at least two patients. We found that both convergence (Fig. $4 G$ ) and divergence (Fig. $4 H$ ) rates were high in parts of the dorsolateral prefrontal cortex (BA46 and particularly in BA8) and in the ventrolateral prefrontal cortex (BA44 and BA45), whereas it was low in the frontopolar cortex and adjacent areas (BA9 and BA10). The grids in Pts. 4 and 6 covered primary sensory and motor areas, respectively (BA2 in Pt. 4 and BA4 in Pt. 6), where the occurrence of convergent and divergent activity was the lowest. In Pt. 4, variable rates of convergence and divergence were found in the middle-superior temporal area and adjacent parietal regions (BA21, BA22, BA39, BA40, BA41, BA42).

The high correlation between input and output patterns of convergence-divergence centers found in this report suggests the presence of reciprocal and short-range circular propagation of SWA. We tested these possibilities as follows. For each unitary propagation event of SWA between two cortical recording sites, we investigated whether reciprocal propagation between those areas appears within $100 \mathrm{~ms}$. The number of reciprocal associations was highly above chance in all analyzed sleep episodes of all subjects ( $\mathrm{p} \approx 0$ in comparison with control distributions) (Fig. $6 A$ ). Finally, three-point circles (i.e., directed three-point trajectories of which the end points are the same as the starting points) of SWA propagation were detected. Three-point circles were abundant in all analyzed epochs (Fig. $6 \mathrm{~B}$ ), showing that propagating SWA often reenters previously visited cortical areas within a short time interval.

\section{Discussion}

Here we analyzed the ECoG patterns of sleep slow waves in humans using a nonlinear correlation technique based on mutual information calculations supplemented by linear correlation measures. While high-density scalp EEG provides nearly complete spatial coverage over both hemispheres with a spatial resolution of $\sim 2 \mathrm{~cm}$ at the level of the scalp, its localizing capacity is hampered by the spatial smoothing effects of the scalp, skull, pia, and CSF. In contrast, while ECoG recordings provide lower spatial coverage, with a higher spatial resolution of $\sim 1 \mathrm{~cm}$ at the level of the cortical surface, it is not burdened by the spatial smoothing effects of the interleaving tissue (Buzsaki, 2006).

Correlations among SWA cycles recorded from different ECoG channels were highest during the first part of the up states where 
A
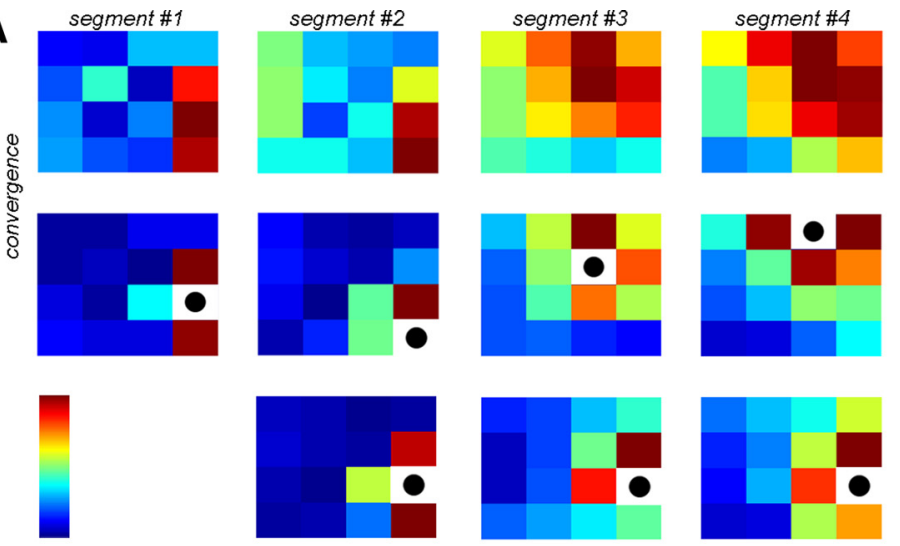

B
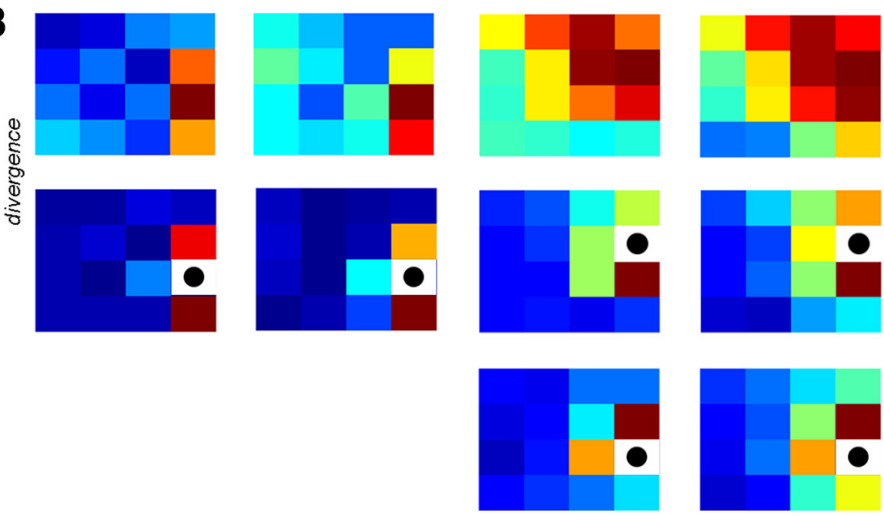

Figure 5. Convergence and divergence maps were similar within patients across sleep segments. $\boldsymbol{A}$, Top, Convergence maps of Pt. 3 (see color bar). First and second segments were recorded during the second postoperative night; third and fourth segments are from the fourth postoperative night; fifth segment was recorded during the fifth postoperative night Middle, Relative contribution to convergent inputs to the area with highest convergence rate. Bottom, Relative contribution to convergent inputs of the site receiving the most convergent activity during the first segment. $\boldsymbol{B}$, Divergence maps and relative distribution of diverging activities arranged similarly as convergence maps in $\boldsymbol{A}$. Note that maps for SWS segments recorded during a single night (first and second; third and fourth) were markedly similar in all cases. Maps in the fifth column are clearly similar to those of the third and fourth segments, despite being recorded during two different (but consecutive) nights.

A

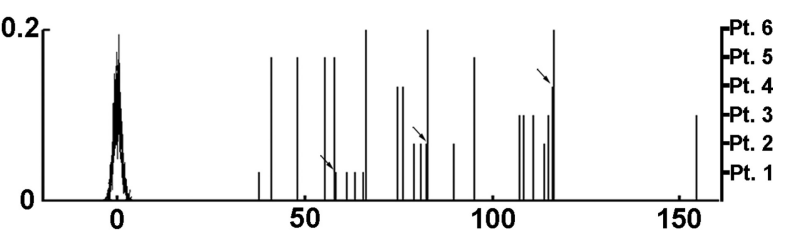

B 0.2

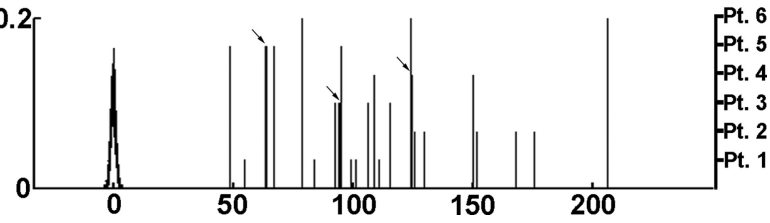

Figure 6. Reciprocal connections and short circles in the course of SWA propagation. $\boldsymbol{A}$, Normalized number of reciprocal connections calculated for all analyzed sleep segments in all subjects. Normalization was performed using control distributions (see Materials and Methods). Normalized control distributions are superimposed in black (left $y$-axis shows probabilities). Normalized numbers of reciprocal propagations are indicated as vertical bars (different lengths correspond to different patients; right $y$-axis). The number of reciprocal connections was found to be highly above control values in all cases $(p \approx 0)$. Arrows point to overlapping values. $\boldsymbol{B}$, Normalized number of three-point circles displayed in a similar way as reciprocal connections. The number of circles was significantly above control values $(p \approx 0)$.
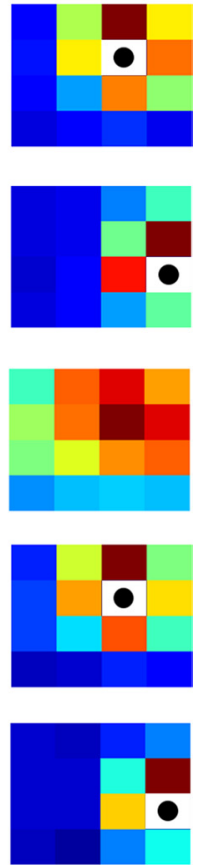

most of the neuronal spiking interactions take place, confirming that this phase of the oscillation provides an optimal window for neuronal interactions (Luczak et al., 2009). It is also in line with previous findings showing that sleep spindles and hippocampal ripples are phase locked to this segment of the slow-wave cycle, indicating that the flow of information both among cortical regions and between the thalamocortical and hippocampal circuits would be connected to the down-to-up state transition period (Mölle et al., 2006; Clemens et al., 2007).

SWA, recorded by ECoG, showed complex propagation patterns such as convergence, divergence, reciprocal propagation, and short circles. Correlations were strongest between neighboring recording sites, although unexpected spatial noncontinuities of slow-wave propagation were also observed. Cortical areas were shown to receive convergent activity from other regions as well as to disseminate SWA. Centers (or hot spots) of SWA propagation with high convergence and divergence rates were identified in all subjects. Hot spots were stable during and, although to a lesser extent, across nights. These complex patterns of SWA propagation might reflect the differential aspects of local and global mechanisms of neural interactions revealed by cortical electrical activity during SWS.

Our choice of the nonlinear correlation measure was based on our observation and other reports that, in addition to linear associations, nonlinear dependencies are frequently detected among different brain regions during various brain states (Freiwald et al., 1999; Darvas et al., 2009; Chen et al., 2010). Although linear methods (e.g., cross-correlation, partial directed coherence, directed transfer function) may in certain situations perform better than nonlinear ones (e.g., MI, transfer entropy, nonlinear Granger causality), they has common drawbacks, such as bias toward the detection of linear associations and a lack of scaling independence (for review, see Pereda et al., 2005). The well established information theory-based MI analysis (Na et al., 2002; Kajikawa and Hackett, 2005; Pereda et al., 2005; Paz et al., 2010) was capable of detecting a larger number of significant associations when compared with linear cross-correlations. These nonlinear associations were often characterized by complex and variable input-output functions. Our choices of crosscorrelation and MI were in concert with the recommendations of Pereda et al. (2005) for EEG-type data. Functional connections are inferred from linear or nonlinear correlation or coherence measures, such as significant short-latency peaks in crosscorrelation functions when established between single neurons (Csicsvari et al., 1998; Barthó et al., 2004; Hangya et al., 2010) or significant waveform and phase correlations in cases of neural networks or brain areas (Buzsaki, 2006; Womelsdorf et al., 2007; Bullmore and Sporns, 2009; Singer and Uhlhaas et al., 2009). Thus, we speculate that nonlinear correlation of SWA activity might reflect functional connections among brain areas. 
Associations reported in this study may be mediated by both corticocortical and corticothalamocortical routes of information transmission (Crunelli and Hughes, 2010). Although it is theoretically possible that the two types of communication operate with different temporal delays, consistent bimodality of propagation time distributions could not be revealed. Also, influences of common information sources cannot completely be ruled out (Hangya et al., 2009), although a recent study suggested that such sources do not necessarily cause detectable correlations (Renart et al., 2010).

Based on the concept of traveling waves, a propagating slow wave rarely if ever reenters previously visited cortical sites. However, anatomical studies of cortical structural connectivity show an especially rich repertoire of reciprocal connections and loops within and across brain areas (Bullmore and Sporns, 2009). In concert with the complex patterns of cortical connectivity, we also found a rich source of reciprocal and short circle propagation patterns, which may suggest that electrical activity underlying neuronal processing was often bidirectional, or reentered previously visited areas.

We propose that cortical centers with high convergence and divergence rates might serve as hot spots for neuronal interaction in sleep. This view is supported by the relative stability of these hot spots and their tendency to have a larger correlation with neighboring cortical areas. Spatial distances between correlated channels were found to be short on average, which further emphasizes the importance of the finely structured, local neuronal circuitry in electrical activity pattern generation. Longer range correlations arose occasionally, presumably as a consequence of the communication between high connectivity centers. These associations could appear as noncontinuous propagation between relatively distant areas. It should be noted, however, that rapid step-by-step spread along a continuous array of recording sites could be masked sometimes by higher noise levels on some of the electrodes or reduced signal quality over sulci. Between-night correlations appeared less frequently compared with withinnight correlations, which may be a result of slow temporal changes in cortical activity. This hypothesis is in agreement with previous results showing that local changes in cortical SWA are largely related to daytime experiences (Huber et al., 2004). We did not observe a systematic change of correlation stability with increasing time after the surgery. However, an effect of gradual recovery after the surgical intervention on the stability of convergence and divergence centers cannot be completely ruled out.

Although different placements of the electrode grid on the six patients limited the possibilities of mapping hot spots onto anatomical structures, averaging normalized convergence and divergence rates in different Brodmann areas showed an elevated activity in the ventrolateral and dorsolateral prefrontal cortex. These observations are in accordance with previous studies showing the strong involvement of human prefrontal areas in memory consolidation and related delta band oscillatory activity during deep stages of sleep (Diekelmann and Born, 2010). Our findings are also in agreement with the prominent participation of the middle and inferior frontal gyri in SWS propagation (Murphy et al., 2009).

The long-standing observation that sleep is beneficial to remembering (Jenkins and Dallenbach, 1924) has recently been linked to cortical sleep slow waves (Fowler et al., 1973; Marshall et al., 2006). Although large brain areas can engage in synchronous low-frequency oscillations (Sirota and Buzsáki, 2005; Volgushev et al., 2006), a number of studies showed localized increase of SWA after learning correlated with memory performance after sleep (Huber et al., 2004; Schmidt et al., 2006; Huber et al., 2007; Massimini et al., 2009 and references therein). Thus, a growing body of evidence suggests that memory consolidation during sleep involves localized cortical processes, which are accompanied by regional changes in SWA. In addition to reports of SWA propagation over wide cortical areas (Massimini et al., 2004; Hill and Tononi, 2005; Murphy et al., 2009), propagation dynamics should be examined at a finer spatial scale to detect phenomena possibly underlying local cortical processing (Luczak et al., 2007; Mohajerani et al., 2010). The use of subdural electrode grids, which lack the major drawbacks of scalp recordings, such as spatial smoothing and attenuation exerted by the intermediate tissues (Buzsaki, 2006; Bangera et al., 2010), allowed us to unravel an unexpected complexity of localized SWA propagation patterns, e.g., short circles and convergence-divergence centers. Thus, our results provide the first step in filling the gap between global SWA traveling (Massimini et al., 2004) and local operations of cortical networks (Huber et al., 2004; Mohajerani et al., 2010).

\section{Notes}

Supplemental material for this article is available at http://www.koki.hu/ supp_materials/Hangya_et_al_Supplemental_Material_JN2011.html. The supplemental material includes three movies showing slow oscillation propagation (two for real and one for simulated data); additional analysis showing robustness of our results, including an analysis of signal-to-noise ratios with three figures; and three tables showing individual propagation speed and convergence/divergence map correlation results, as well as coverage of Brodmann areas by the electrode grids for each patient. This material has not been peer reviewed.

\section{References}

Bangera NB, Schomer DL, Dehghani N, Ulbert I, Cash S, Papavasiliou S, Eisenberg SR, Dale AM, Halgren E (2010) Experimental validation of the influence of white matter anisotropy on the intracranial EEG forward solution. J Comput Neurosci 29:371-387.

Barthó P, Hirase H, Monconduit L, Zugaro M, Harris KD, Buzsáki G (2004) Characterization of neocortical principal cells and interneurons by network interactions and extracellular features. J Neurophysiol 92:600-608.

Bullmore E, Sporns O (2009) Complex brain networks: graph theoretical analysis of structural and functional systems. Nat Rev Neurosci 10:186-198.

Buzsaki G (2006) Rhythms of the brain. New York: Oxford University.

Caporale N, Dan Y (2008) Spike timing-dependent plasticity: a Hebbian learning rule. Annu Rev Neurosci 31:25-46.

Cash SS, Halgren E, Dehghani N, Rossetti AO, Thesen T, Wang C, Devinsky O, Kuzniecky R, Doyle W, Madsen JR, Bromfield E, Eross L, Halász P, Karmos G, Csercsa R, Wittner L, Ulbert I (2009) The human K-complex represents an isolated cortical down-state. Science 324:1084-1087.

Chen CC, Kilner JM, Friston KJ, Kiebel SJ, Jolly RK, Ward NS (2010) Nonlinear coupling in the human motor system. J Neurosci 30:8393-8399.

Clemens Z, Mölle M, Eross L, Barsi P, Halász P, Born J (2007) Temporal coupling of parahippocampal ripples, sleep spindles and slow oscillations in humans. Brain 130:2868-2878.

Cossart R, Aronov D, Yuste R (2003) Attractor dynamics of network UP states in the neocortex. Nature 423:283-288.

Crunelli V, Hughes SW (2010) The slow $(<1 \mathrm{~Hz})$ rhythm of non-REM sleep: a dialogue between three cardinal oscillators. Nat Neurosci 13:9-17.

Csercsa R, Dombovári B, Fabó D, Wittner L, Eross L, Entz L, Sólyom A, Rásonyi G, Szucs A, Kelemen A, Jakus R, Juhos V, Grand L, Magony A, Halász P, Freund TF, Maglóczky Z, Cash SS, Papp L, Karmos G, et al. (2010) Laminar analysis of slow wave activity in humans. Brain 133:2814-2829.

Csicsvari J, Hirase H, Czurko A, Buzsáki G (1998) Reliability and state dependence of pyramidal cell-interneuron synapses in the hippocampus: an ensemble approach in the behaving rat. Neuron 21:179-189.

Darvas F, Ojemann JG, Sorensen LB (2009) Bi-phase locking-a tool for 
probing non-linear interaction in the human brain. Neuroimage 46:123-132.

Diekelmann S, Born J (2010) The memory function of sleep. Nat Rev Neurosci 11:114-126.

Fowler MJ, Sullivan MJ, Ekstrand BR (1973) Sleep and memory. Science 179:302-304.

Freiwald WA, Valdes P, Bosch J, Biscay R, Jimenez JC, Rodriguez LM, Rodriguez V, Kreiter AK, Singer W (1999) Testing non-linearity and directedness of interactions between neural groups in the macaque inferotemporal cortex. J Neurosci Methods 94:105-119.

Hangya B, Borhegyi Z, Szilágyi N, Freund TF, Varga V (2009) GABAergic neurons of the medial septum lead the hippocampal network during theta activity. J Neurosci 29:8094-8102.

Hangya B, Li Y, Muller RU, Czurkó A (2010) Complementary spatial firing in place cell-interneuron pairs. J Physiol 588:4165-4175.

Hill S, Tononi G (2005) Modeling sleep and wakefulness in the thalamocortical system. J Neurophysiol 93:1671-1698.

Huang X, Xu W, Liang J, Takagaki K, Gao X, Wu JY (2010) Spiral wave dynamics in neocortex. Neuron 68:978-990.

Huber R, Ghilardi MF, Massimini M, Tononi G (2004) Local sleep and learning. Nature 430:78-81.

Huber R, Esser SK, Ferrarelli F, Massimini M, Peterson MJ, Tononi G (2007) TMS-induced cortical potentiation during wakefulness locally increases slow wave activity during sleep. PLoS One 2:e276.

Iber C, Ancoli-Israel S, Chesson A, Quan SF (2007) The AASM manual for the scoring of sleep and associated events: rules, terminology and technical specifications. Westchester: American Academy of Sleep Medicine.

Isomura Y, Sirota A, Ozen S, Montgomery S, Mizuseki K, Henze DA, Buzsáki G (2006) Integration and segregation of activity in entorhinal-hippocampal subregions by neocortical slow oscillations. Neuron 52:871-882.

Jenkins JC, Dallenbach KM (1924) Obliviscence during sleep and waking. Am J Psychol 35:605-612.

Kajikawa Y, Hackett TA (2005) Entropy analysis of neuronal spike train synchrony. J Neurosci Methods 149:90-93.

Kurth S, Ringli M, Geiger A, LeBourgeois M, Jenni OG, Huber R (2010) Mapping of cortical activity in the first two decades of life: a high-density sleep electroencephalogram study. J Neurosci 30:13211-13219.

Le Van Quyen M, Staba R, Bragin A, Dickson C, Valderrama M, Fried I, Engel J (2010) Large-scale microelectrode recordings of high-frequency gamma oscillations in human cortex during sleep. J Neurosci 30:7770-7782.

Luczak A, Barthó P, Marguet SL, Buzsáki G, Harris KD (2007) Sequential structure of neocortical spontaneous activity in vivo. Proc Natl Acad Sci U S A 104:347-352.

Luczak A, Barthó P, Harris KD (2009) Spontaneous events outline the realm of possible sensory responses in neocortical populations. Neuron 62:413-425.

Magnin M, Rey M, Bastuji H, Guillemant P, Mauguière F, Garcia-Larrea L (2010) Thalamic deactivation at sleep onset precedes that of the cerebral cortex in humans. Proc Natl Acad Sci U S A 107:3829-3833.

Marshall L, Helgadóttir H, Mölle M, Born J (2006) Boosting slow oscillations during sleep potentiates memory. Nature 444:610-613.

Massimini M, Huber R, Ferrarelli F, Hill S, Tononi G (2004) The sleep slow oscillation as a traveling wave. J Neurosci 24:6862-6870.

Massimini M, Tononi G, Huber R (2009) Slow waves, synaptic plasticity and information processing: insights from transcranial magnetic stimulation and high-density EEG experiments. Eur J Neurosci 29:1761-1770.
Mena-Segovia J, Sims HM, Magill PJ, Bolam JP (2008) Cholinergic brainstem neurons modulate cortical gamma activity during slow oscillations. J Physiol 586:2947-2960.

Mohajerani MH, McVea DA, Fingas M, Murphy TH (2010) Mirrored bilateral slow-wave cortical activity within local circuits revealed by fast bihemispheric voltage-sensitive dye imaging in anesthetized and awake mice. J Neurosci 30:3745-3751.

Mölle M, Yeshenko O, Marshall L, Sara SJ, Born J (2006) Hippocampal sharp wave-ripples linked to slow oscillations in rat slow-wave sleep. J Neurophysiol 96:62-70.

Murphy M, Riedner BA, Huber R, Massimini M, Ferrarelli F, Tononi G (2009) Source modeling sleep slow waves. Proc Natl Acad Sci U S A 106:1608-1613.

Na SH, Jin SH, Kim SY, Ham BJ (2002) EEG in schizophrenic patients: mutual information analysis. Clin Neurophysiol 113:1954-1960.

Ostojic S, Brunel N, Hakim V (2009) How connectivity, background activity, and synaptic properties shape the cross-correlation between spike trains. J Neurosci 29:10234-10253.

Panzeri S, Senatore R, Montemurro MA, Petersen RS (2007) Correcting for the sampling bias problem in spike train information measures. J Neurophysiol 98:1064-1072.

Paz R, Gelbard-Sagiv H, Mukamel R, Harel M, Malach R, Fried I (2010) A neural substrate in the human hippocampus for linking successive events. Proc Natl Acad Sci U S A 107:6046-6051.

Pereda E, Quiroga RQ, Bhattacharya J (2005) Nonlinear multivariate analysis of neurophysiological signals. Prog Neurobiol 77:1-37.

Renart A, de la Rocha J, Bartho P, Hollender L, Parga N, Reyes A, Harris KD (2010) The asynchronous state in cortical circuits. Science 327:587-590.

Schmidt C, Peigneux P, Muto V, Schenkel M, Knoblauch V, Münch M, de Quervain DJ, Wirz-Justice A, Cajochen C (2006) Encoding difficulty promotes postlearning changes in sleep spindle activity during napping. J Neurosci 26:8976-8982.

Shannon C (1948) A mathematical theory of communication. Bell Syst Tech J 27:379- 423, 623-656.

Siapas AG, Wilson MA (1998) Coordinated interactions between hippocampal ripples and cortical spindles during slow-wave sleep. Neuron 21:1123-1128.

Sirota A, Buzsáki G (2005) Interaction between neocortical and hippocampal networks via slow oscillations. Thalamus Relat Syst 3:245-259.

Steriade M, Nuñez A, Amzica F (1993) A novel slow ( $<1 \mathrm{~Hz}$ ) oscillation of neocortical neurons in vivo: depolarizing and hyperpolarizing components. J Neurosci 13:3252-3265.

Uhlhaas PJ, Pipa G, Lima B, Melloni L, Neuenschwander S, Nikolić D, Singer W (2009) Neural synchrony in cortical networks: history, concept and current status. Front Integr Neurosci 3:17.

Volgushev M, Chauvette S, Mukovski M, Timofeev I (2006) Precise longrange synchronization of activity and silence in neocortical neurons during slow-wave oscillations. J Neurosci [Erratum (2006) 26:table of contents] 26:5665-5672.

Wolansky T, Clement EA, Peters SR, Palczak MA, Dickson CT (2006) Hippocampal slow oscillation: a novel EEG state and its coordination with ongoing neocortical activity. J Neurosci 26:6213-6229.

Womelsdorf T, Schoffelen JM, Oostenveld R, Singer W, Desimone R, Engel AK, Fries P (2007) Modulation of neuronal interactions through neuronal synchronization. Science 316:1609-1612. 